EPJ Web of Conferences 71, 00115 (2014)

DOI: 10.1051/epjconf/20147100115

(C) Owned by the authors, published by EDP Sciences, 2014

\title{
PAMELA mission: heralding a new era in cosmic ray physics
}

S. B. Ricciarini ${ }^{1, a}$, O. Adriani ${ }^{1,2}$, G.C. Barbarino ${ }^{3,4}$, G.A. Bazilevskaya ${ }^{5}$, R. Bellotti6,7, M. Boezio ${ }^{8}$, E.A. Bogomolov ${ }^{9}$, M. Bongi ${ }^{1,2}$, V. Bonvicini ${ }^{8}$, S. Bottai ${ }^{1}$, A. Bruno ${ }^{6,7}$, F. Cafagna ${ }^{7}$, D. Campana ${ }^{4}$, R. Carbone ${ }^{8}$, P. Carlson ${ }^{10}$, M. Casolino ${ }^{11,12}$, G. Castellini' ${ }^{13}$, C. De Donato ${ }^{11}$, M.P. De Pascale ${ }^{11,14,21}$, C. De Santis ${ }^{11,14}$, N. De Simone ${ }^{11}$, V. Di Felice ${ }^{11}$, V. Formato ${ }^{8,15}$, A.M. Galper ${ }^{16}$, A.V. Karelin ${ }^{16}$, M.D. Kheymits ${ }^{16}$, S.V. Koldashov ${ }^{16}$, S. Koldobskiy ${ }^{16}$, S.Yu. Krutkov ${ }^{9}$, A.N. Kvashnin ${ }^{5}$, A. Leonov ${ }^{16}$, V. Malakhov ${ }^{16}$, L. Marcelli ${ }^{14}$, M. Martucci ${ }^{14,17}$, A.G. Mayorov ${ }^{16}$, W. Menn ${ }^{18}$, M. Mergè ${ }^{11,14}$, V.V. Mikhailov ${ }^{16}$, E. Mocchiutti ${ }^{8}$, A. Monaco $^{7}$, N. Mori ${ }^{1}$, R. Munini ${ }^{8,15}$, G. Osteria ${ }^{4}$, F. Palma ${ }^{11,14}$, B. Panico ${ }^{4}$, P. Papini ${ }^{1}$, M. Pearce ${ }^{10}$, P. Picozza ${ }^{11,14}$, C. Pizzolotto ${ }^{19,20,22}$, M. Ricci ${ }^{17}$, R. Sarkar ${ }^{8}$, M. Simon ${ }^{18}$, V. Scotti ${ }^{3,4}$, R. Sparvoli ${ }^{11,14}$, P. Spillantini ${ }^{1,2}$, Y.I. Stozhkov ${ }^{5}$, A. Vacchi ${ }^{8}$, E. Vannuccini ${ }^{1}$, G.I. Vasilyev ${ }^{9}$, S.A. Voronov ${ }^{16}$, Y.T. Yurkin ${ }^{16}$, G. Zampa ${ }^{8}$, N. Zampa ${ }^{8}$, and V.G. Zverev ${ }^{16}$

${ }^{1}$ INFN, Sezione di Florence, I-50019 Sesto Fiorentino, Florence, Italy

${ }^{2}$ University of Florence, Department of Physics, I-50019 Sesto Fiorentino, Florence, Italy

${ }^{3}$ University of Naples "Federico II", Department of Physics, I-80126 Naples, Italy

${ }^{4}$ INFN, Sezione di Naples, I-80126 Naples, Italy

${ }^{5}$ Lebedev Physical Institute, RU-119991, Moscow, Russia

${ }^{6}$ University of Bari, Department of Physics, I-70126 Bari, Italy

${ }^{7}$ INFN, Sezione di Bari, I-70126 Bari, Italy

${ }^{8}$ INFN, Sezione di Trieste, I-34149 Trieste, Italy

${ }^{9}$ loffe Physical Technical Institute, RU-194021 St. Petersburg, Russia

${ }^{10}$ KTH, Department of Physics, and the Oskar Klein Centre for Cosmoparticle Physics, AlbaNova University

Centre, SE-10691 Stockholm, Sweden

${ }^{11}$ INFN, Sezione di Rome "Tor Vergata", I-00133 Rome, Italy

${ }^{12}$ RIKEN, Advanced Science Institute, Wako-shi, Saitama, Japan

${ }^{13}$ IFAC, I-50019 Sesto Fiorentino, Florence, Italy

${ }^{14}$ University of Rome "Tor Vergata", Department of Physics, I-00133 Rome, Italy

${ }^{15}$ University of Trieste, Department of Physics, I-34147 Trieste, Italy

${ }^{16}$ NRNU MEPhl, RU-115409 Moscow, Russia

${ }^{17}$ INFN, Laboratori Nazionali di Frascati, Via Enrico Fermi 40, I-00044 Frascati, Italy

${ }^{18}$ Universitat Siegen, Department of Physics, D-57068 Siegen, Germany

${ }^{19}$ INFN, Sezione di Perugia, I-06123 Perugia, Italy

${ }^{20}$ Agenzia Spaziale Italiana (ASI) Science Data Center, I-00044 Frascati, Italy

${ }^{21}$ Deceased

${ }^{22}$ Previously at INFN, Sezione di Trieste, I-34149 Trieste, Italy

\begin{abstract}
After seven years of data taking in space, the experiment PAMELA is showing very interesting features in cosmic rays, namely in the fluxes of protons, helium, electrons, that might change our basic vision of the mechanisms of production, acceleration and propagation of cosmic rays in the galaxy. In addition, PAMELA measurements of
\end{abstract}

a e-mail: ricciarini@fi.infn.it

This is an Open Access article distributed under the terms of the Creative Commons Attribution License 2.0, which permits unrestricted use, distribution, and reproduction in any medium, provided the original work is properly cited. 


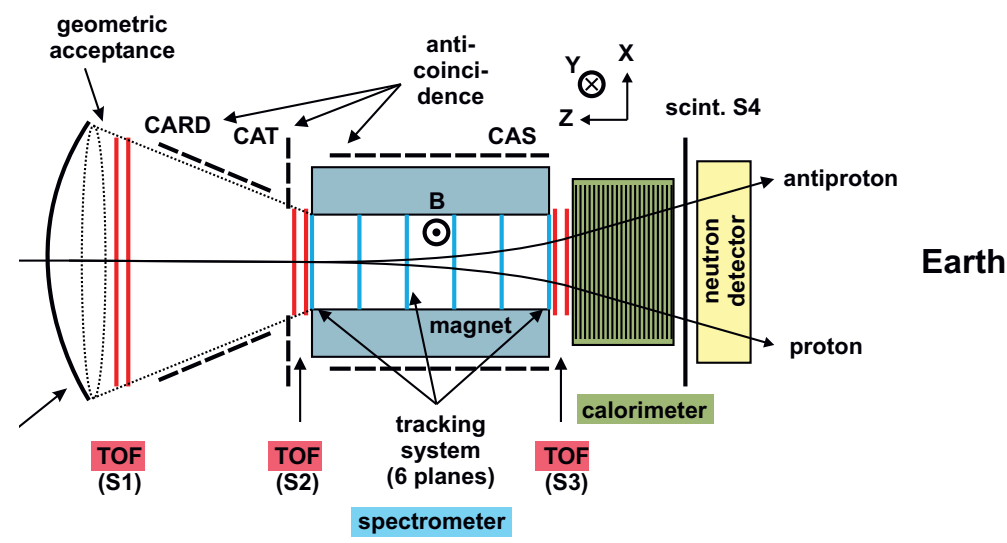

Figure 1. Schematic view of the PAMELA apparatus, with indicated the main direction of the magnetic field (B) inside the spectrometer cavity. PAMELA is $1.3 \mathrm{~m}$ long and less than $1 \mathrm{~m}$ wide.

cosmic antiproton and positron fluxes are setting strong constraints to the nature of Dark Matter. The continuous particle detection is allowing a constant monitoring of the solar activity and detailed study of the solar modulation for a long period, giving important improvements to the comprehension of the heliosphere mechanisms. PAMELA is also measuring the radiation environment around the Earth, and has recently discovered an antiproton radiation belt.

\section{Introduction}

The PAMELA satellite-borne experiment [1] is composed of a set of high-precision cosmic-ray detectors, sensitive to particles and antiparticles with identification of the charge sign, and to light nuclei $(\mathrm{Z}<8)$. It is installed on a low-Earth-orbit ${ }^{1}$ (LEO) satellite and in continuous data acquisition mode since July 2006.

The apparatus is described in Sect. 2. In Sect. 3 we review some of the most significant scientific results obtained with the PAMELA experiment and affecting various research fields, including cosmicray theories, exotic physics and the study of solar and terrestrial environment. Sect. 4 contains some concluding remarks.

\section{PAMELA apparatus and satellite mission}

The PAMELA apparatus (see figure 1) is housed inside a pressurized Al vessel filled with $\mathrm{N}_{2}$ at 1 atm, with thickness of $2 \mathrm{~mm}$ in correspondence of the PAMELA acceptance window, and installed on-board of the Resurs-DK1 satellite, following an orbit with altitude varying between 350 and 600 $\mathrm{km}$ (stabilized at $600 \mathrm{~km}$ since 2010) and inclination of $70^{\circ}$.

The core of the PAMELA apparatus is formed by a high-precision magnetic spectrometer: a 140 $\mathrm{kg}$ permanent magnet produces an approximately uniform and dipolar magnetic field of $\approx 0.4 \mathrm{~T}$ in a $16 \times 13 \times 44 \mathrm{~cm}^{3}$ rectangular cavity, defining a geometrical factor of $21.6 \mathrm{~cm}^{2} \mathrm{sr}$.

\footnotetext{
${ }^{1}$ This term is commonly used to indicate an orbit with altitude below $2000 \mathrm{~km}$.
} 
The spectrometer is equipped with a tracking system composed of six equidistant planes of doublesided microstrip Si detectors, thus providing two independent impact coordinates with uncertainty of 3 and $12 \mu \mathrm{m}$ respectively, for normally incident trajectories. The relative positions of the Si sensors have been determined with an in-flight alignment procedure, using the collected relativistic $\mathrm{p}, \mathrm{e}^{-}$and $\mathrm{e}^{+}$, which had been previously validated at beam tests and characterized by a precision better than 1 $\mu \mathrm{m}$.

The magnetic spectrometer determines the sign of the electric charge $\mathrm{Ze}$ of the incident particle and its magnetic deflection $\eta=\mathrm{Ze} / \mathrm{Pc}$ (where $\mathrm{Pc}$ is the particle momentum in $\mathrm{GeV}$ ) and rigidity $\mathrm{R}=$ $1 /|\eta|$. For relativistic particles, the average rigidity uncertainty follows the law $\delta \mathrm{R} / \mathrm{R}=\mathrm{R} / \mathrm{MDR}$, with MDR (maximum detectable rigidity) defined as the rigidity for which the relative uncertainty is $\delta \mathrm{R} / \mathrm{R}$ $=1$. From measurements at beam tests, the MDR distribution exceeds $1 \mathrm{TV}$.

The time-of-flight (ToF) system comprises three double planes (S1, S2 and S3) of fast plastic scintillators, segmented into paddles with photo-multiplier read-out. Coincidental energy deposits in combinations of layers provide the main trigger for the experiment. ToF information is combined with spectrometer track-length information to determine velocities for non-relativistic particles. The measured ToF resolution, better than $300 \mathrm{ps,} \mathrm{allows} \mathrm{e}^{-} / \mathrm{e}^{+}$to be separated from $\mathrm{p} / \overline{\mathrm{p}}$ up to an energy of $\sim 1 \mathrm{GeV}$. Upward-going particles are rejected by Tof measurement with a significance of 60 standard deviations.

Ionization measurements in the scintillator layers and $\mathrm{Si}$ sensors allow the absolute value of particle charge to be determined at least up to $\mathrm{Z}=7$ and 5 , respectively.

The sampling electromagnetic calorimeter is composed of 22 modules, each one formed by a central plate of W absorber surrounded by 2 single-sided Si strip planes, for a total depth of 16.3 radiation lengths and 0.6 nuclear interaction lengths. The main task of the calorimeter is to discriminate $\mathrm{e}^{+}\left(\mathrm{e}^{-}\right)$from $\mathrm{p}(\overline{\mathrm{p}})$; the longitudinal and transverse segmentation of the calorimeter, combined with the measurement of the energy deposited on each Si strip, allows a high discrimination power between electromagnetic showers and hadron events.

PAMELA achieves a proton rejection power better than $10^{5}$ up to an energy of $\sim 200 \mathrm{GeV}$, with a positron selection efficiency of $80 \%$, when employing suitably strong cuts, based on the topology of the shower inside the calorimeter, and when requiring a match between the total energy release detected in the calorimeter and the particle momentum given by the spectrometer.

The anti-coincidence system consists of four plastic scintillators (CAS) surrounding the sides of the magnet, one (CAT) covering the top and four (CARD) delimiting the volume between the first two ToF planes. The main purpose of this system is to identify events characterized by secondary particles produced in the apparatus.

\section{Selected results of PAMELA experiment}

The present section summarizes the most important results obtained with the PAMELA apparatus up to now, determined by considering the impact of each measurement on the affected theoretical models. A description of the methods employed to perform each single measurement, and to evaluate the associated uncertainties, is beyond the scope of the present paper: detailed information can be found within the PAMELA publications cited throughout this section.

\subsection{Cosmic-ray hydrogen and helium spectra}

The PAMELA collaboration published [2] the first ever high-precision measurements of the absolute fluxes of galactic hydrogen (mostly protons) and helium, in the rigidity range $1 \mathrm{GV}$ to $1.2 \mathrm{TV}$, characterized by a total uncertainty of few $\%$ on single data points. Such high precision allowed for the 


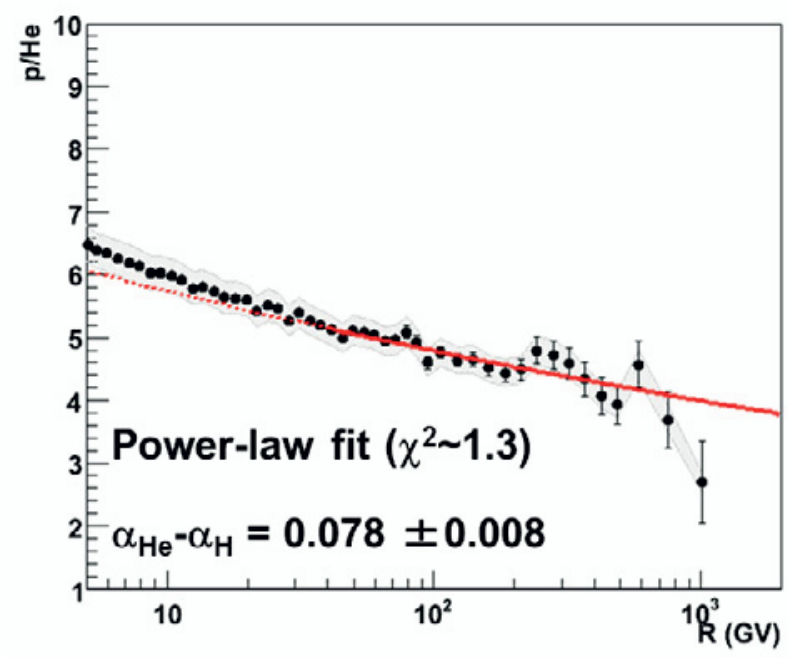

Figure 2. The ratio between $\mathrm{H}$ (mostly protons) and He fluxes measured with PAMELA [2]. The gray shaded area represents the measurement systematic uncertainty. The figure also shows the result of a power-law fit which takes into account data points above $40 \mathrm{GV}$.

discovery of fine deviations of the measured rigidity spectra from the standard assumption, i.e. that both fluxes are well described by a single power law with intensity $\propto R^{-\alpha}$ (with $\mathrm{R}$ particle rigidity) and same spectral index $\alpha \approx 2.7$, thus challenging the current understanding of cosmic-ray acceleration and propagation mechanisms.

The first striking feature, which can be found in the PAMELA spectra, is that $\mathrm{H}$ and $\mathrm{He}$ are characterized by different spectral indexes; this behaviour can be clearly seen in figure 2 , showing the ratio of $\mathrm{H}$ and $\mathrm{He}$ fluxes. The spectral index of $\mathrm{He}$ is higher than the one of $\mathrm{H}$, by $\approx 0.08$.

Besides, the measured spectra show deviations from a single-power-law behaviour, even taking into account the worst-case combination of the estimated systematic errors associated to the measurement:

- both $\mathrm{H}$ and $\mathrm{He}$ spectra gradually soften ${ }^{2}$ in the rigidity range from 30 to $230 \mathrm{GV}$; e.g. for $\mathrm{H}$, a spectral index $\alpha=2.80 \pm 0.01$ is given by a fit to the data points in the range 30 to $80 \mathrm{GV}$, while in the range 80 to $230 \mathrm{GV}, \alpha=2.85 \pm 0.02$;

- an abrupt spectral hardening is found at rigidity $\mathrm{R} \approx 235 \mathrm{GV}$, with the spectral index $\alpha$ decreasing of $\approx 0.2$ for $\mathrm{H}$ (from 2.85 to 2.67 ) and $\approx 0.3$ for $\mathrm{He}$ (from 2.77 to 2.48 ).

By considering the whole set of $\mathrm{H}$ or He data points above $30 \mathrm{GV}$, a single-power-law fit is rejected at $98 \%$ confidence level. This conclusion is in broad agreement with results from previous experiments, performed at higher energies than PAMELA and with lower precision, as discussed in [2].

The PAMELA H and He measurements have triggered a critical review of the standard theory of acceleration and propagation of cosmic rays in the galaxy, in an attempt to reproduce PAMELA data points by tuning or better approximating several aspects of the models.

\footnotetext{
${ }^{2}$ Throughout this paper, the term hard (soft) indicates a more (less) intense particle flux, according to the usual terminology.
} 
Various theoretical explanations have been proposed for the observed difference between $\mathrm{H}$ and He spectral indexes: for example, effects of spallation reactions of higher-Z nuclei with interstellar gas during cosmic-ray propagation [3], or acceleration effects, namely differences between the two species in the injection phase of the diffusive shock acceleration at supernova remnant (SNR) sites [4].

For what concerns the deviations from single-power-law spectra, observed for both $\mathrm{H}$ and $\mathrm{He}$, several possibilities have been identified (see e.g. [5] for a comprehensive study): the presence, besides SNR's, of additional cosmic-ray sources and acceleration mechanisms, e.g. novae or explosions in superbubbles; secondary effects of the complex propagation mechanisms in the galaxy, not properly taken into account in the models; effects of the discrete space-time distribution of SNR's; presence of nearby SNR's contributing to most of the observed flux.

Recently the AMS-02 collaboration published [6] [7] measurements of $\mathrm{H}$ and He spectra of comparable high precision, showing significant discrepancies with respect to the spectra obtained with PAMELA. A more precise understanding of these discrepancies will be possible as soon as AMS-02 collaboration will publish detailed information on the measured values and uncertainties of single data points and resulting spectral indexes.

\subsection{Cosmic-ray antiparticles: positrons and antiprotons}

The PAMELA antiparticle measurements are implying strong consequences in cosmic-ray physics, astrophysics, cosmology and exotic particle theories. In fact, PAMELA $\mathrm{e}^{+}$measurements challenge the standard model of cosmic-ray antiparticles, which assumes that they are of secondary ${ }^{3}$ origin; on the other hand, the PAMELA $\overline{\mathrm{p}}$ measurements put very strict constraints on non-standard models, especially those involving dark matter.

\subsubsection{PAMELA positrons}

In 2009 the PAMELA collaboration reported [8] the first ever clear evidence of an excess in the cosmic-ray positron fraction, i.e. the flux ratio $\mathrm{F}\left(\mathrm{e}^{+}\right) / \mathrm{F}\left(\mathrm{e}^{+}+\mathrm{e}^{-}\right)$, for energies above $\approx 10 \mathrm{GeV}$.

Attempts, made to review assumptions and approximations in the standard description of cosmicray propagation (see for example [9] [10]), were not completely satisfying in reproducing PAMELA data points; on the other hand, the PAMELA excess can be explained by introducing an additional primary source, characterized by a harder spectrum for $\mathrm{e}^{+}$than for $\mathrm{e}^{-}$. Various models have been proposed in several hundreds of theoretical papers (see e.g. [11] [12] [13] [14] [15]), where the proposed primary sources can be schematically summarized as: nearby astrophysical sources, such as $\mathrm{e}^{+} \mathrm{e}^{-}$pair production in pulsar magnetosphere; secondary production and re-acceleration in the SNR environment; exotic sources, in particular dark-matter decay or annihilation in the galactic halo.

The high-energy excess found with PAMELA has been later confirmed by Fermi and subsequently AMS-02 experiments, the latter introducing a significant improvement in statistical precision (see [16] and references therein).

The PAMELA collaboration has recently published [16] a new measurement of the positron fraction in the energy range from 1.5 to $300 \mathrm{GeV}$, confirming their previous results; this analysis has been performed on the whole data set collected since launch throughout the whole 2009, and by employing multivariate classification algorithms, thus achieving an overall increase in statistics of about $250 \%$.

\footnotetext{
${ }^{3}$ Throughout this paper, following the usual terminology, we indicate as primary cosmic-ray particles those generated by primary processes (such as acceleration at SNR sites), as secondary cosmic-ray particles those generated via interactions of primary cosmic-rays and interstellar gas.
} 


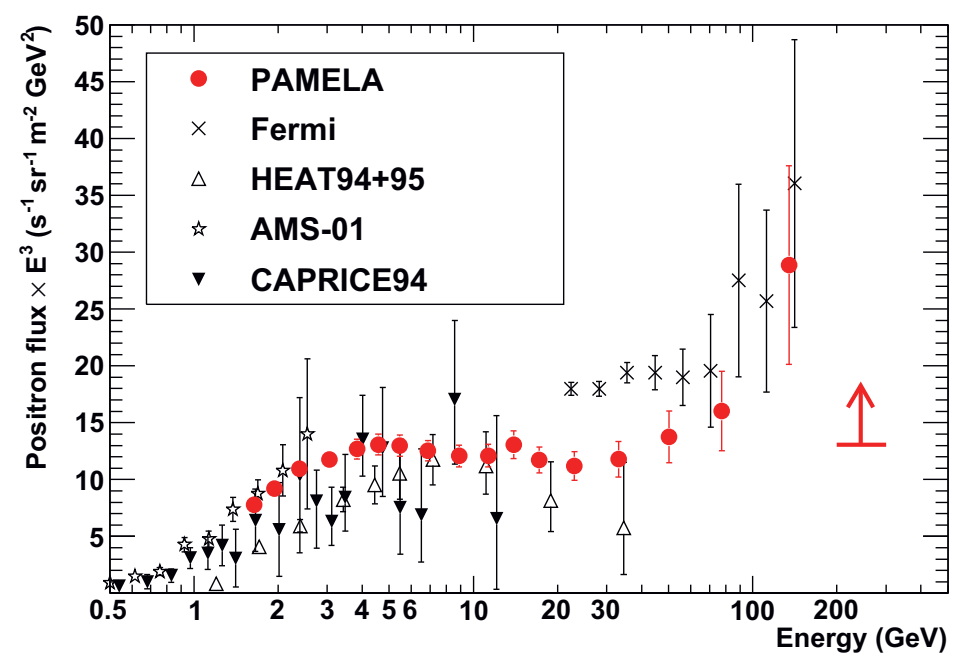

Figure 3. Measurement of absolute positron flux obtained by PAMELA and other experiments, as published in [16].

In the same paper, the PAMELA measurement of the absolute $\mathrm{e}^{+}$flux has been presented for the first time (see figure 3): the $\mathrm{e}^{+}$flux is an important additional tool to understand the theoretical models of $\mathrm{e}^{+}$primary sources, since it sets more stringent limits on model parameters, than those obtained with the positron fraction.

\subsubsection{PAMELA antiprotons}

High-precision measurements of the $\overline{\mathrm{p}}$ flux and $\overline{\mathrm{p}} / \mathrm{p}$ flux ratio have been published [17] [18] by the PAMELA collaboration, covering the kinetic energy range from $60 \mathrm{MeV}$ to $180 \mathrm{GeV}$. These data allow for a powerful discrimination between different models of secondary generation and propagation of $\bar{p}$ in the galaxy, and for fine adjustment of the model parameters.

Besides, it has been shown [19] that these measurements constitute a fundamental tool to exclude or strongly constrain most dark-matter annihilation and decay models of $\mathrm{e}^{+}$production, by probing large regions of the parameters space, with similar sensitivity to the reference gamma-ray observations made by Fermi experiment.

\subsection{Cosmic-ray light-nuclei isotopes}

Precise measurements of secondary/primary flux ratios for light nuclei and their isotopes, are very important for correctly tuning models of cosmic-ray propagation in the galaxy, since the secondary/primary ratio, as locally measured in the solar system, is sensitive to the average amount of traversed matter from the SNR acceleration sites. It has also been pointed out [20] that ${ }^{2} \mathrm{H} /{ }^{1} \mathrm{H}$ and ${ }^{3} \mathrm{He} /{ }^{4} \mathrm{He}$ flux ratios are complementary to the classic $\mathrm{B} / \mathrm{C}$ measurement, in constraining propagation models.

The PAMELA collaboration recently published [21] a high-precision measurement of the ${ }^{2} \mathrm{H} /{ }^{1} \mathrm{H}$ ratio in the kinetic energy range from 100 to $600 \mathrm{MeV} /$ nucleon (shown in figure 4) and of the ${ }^{3} \mathrm{He} /{ }^{4} \mathrm{He}$ 


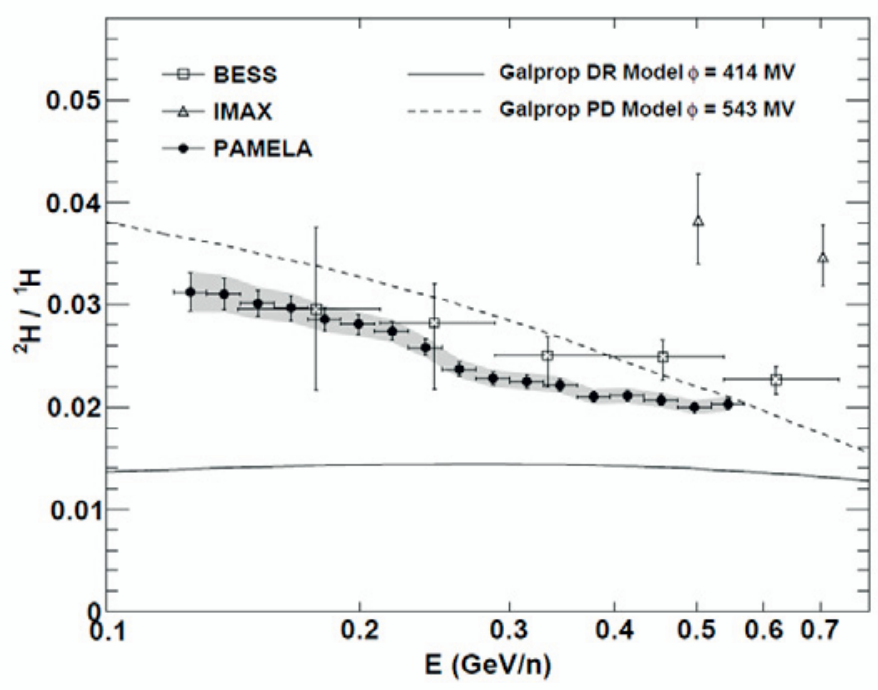

Figure 4. Measurements of ${ }^{2} \mathrm{H} /{ }^{1} \mathrm{H}$ flux ratio by PAMELA and other experiments (see [21] for references), compared with theoretical expectations for different typical galactic propagation models: a DR model (diffusive re-acceleration from irregularities of galactic magnetic field) and a PD model (plain, namely coherent, diffusion only), obtained with GALPROP code [22] with different solar modulation parameter $\phi$.

ratio between 100 and $900 \mathrm{MeV} /$ nucleon, thus obtaining a major improvement with respect to previously available data. For such low-energies, the effect of solar modulation on the observed flux ratio has to be correctly taken into account (see also Sect. 3.4).

\subsection{Solar modulation of cosmic rays}

The PAMELA instrument continuously performed unique multi-particle cosmic-ray observations over several years (from June 2006 to December 2009) of unusually long solar activity minimum, characterized by negative polarity of solar magnetic field, $\mathrm{A}<0$. The collected data constitute a major contribution to the understanding of the various modulation mechanisms (convection, drift, diffusion, adiabatic change) which affect the incoming fluxes of relatively low-energy cosmic-ray particles in the solar system.

The PAMELA collaboration already published [23] a specific paper describing the evolution of the solar modulation for the cosmic-ray proton flux, with precision measurements of the p energy spectra, down to $400 \mathrm{MV}$ rigidity, made during different time periods, thus allowing for fine adjustment of three-dimensional fluido-dynamic models of heliosphere, as shown in figure 5.

Moreover (see for example [24]), the high-precision PAMELA measurements of the low-energy $\overline{\mathrm{p}} / \mathrm{p}$ flux ratio and positron fraction, reported in Sect. 3.2, allow for fine tuning of the theoretical description of charge-sign dependence in the solar modulation, enhanced or suppressed according to the polarity A of the solar magnetic field and to the amount of solar activity. 


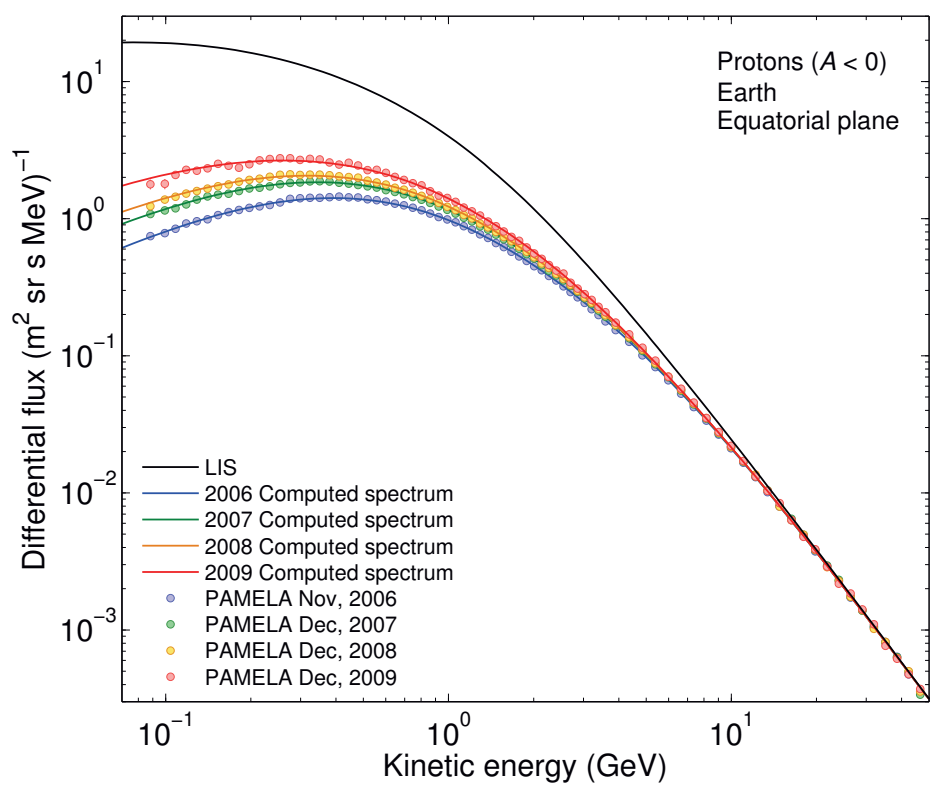

Figure 5. Proton fluxes measured by PAMELA and corresponding tuned spectra from a solar modulation model (figure taken from [23]: see there for information on the employed model). The local interstellar spectrum (LIS) used for the model computation is also shown.

\subsection{Solar energetic particles (SEP)}

The PAMELA apparatus is capable of performing direct SEP measurements in a relatively high kinetic energy range (from $80 \mathrm{MeV} /$ nucleon up to relativistic energies), thus complementing both direct measurements at lower energies, made by dedicated satellites in space, and high-energy indirect measurements, performed on ground by neutron monitors and atmospheric shower detectors, but only possible for the subset of SEP events characterized by accompanying induced ground-level events (GLE).

The PAMELA collaboration published [25] measurements of protons (see figure 6) and He fluxes during the SEP event of 13 December, 2006; these measurements are the first ever direct observation of relativistic SEP. The SEP fluxes add up to the galactic cosmic-ray spectra registered during quiet sun, labelled as "Nov. 2006" in figure 6. In the same figure, it is also possible to see the expected Forbush decrease of galactic particle fluxes, related to the delayed arrival of the coronal mass ejection (CME) accompanying the SEP emission: this effect is visible in the last time slice (14/1650 - 14/2235 UT).

\subsection{Cosmic rays in the Earth magnetosphere}

The PAMELA experiment is producing important contributions to the understanding of the Earth magnetosphere, of the trapping mechanisms of charged particles forming the Van Allen radiation belts, of their interactions with the underlying atmosphere. This information is fundamental for the knowledge 


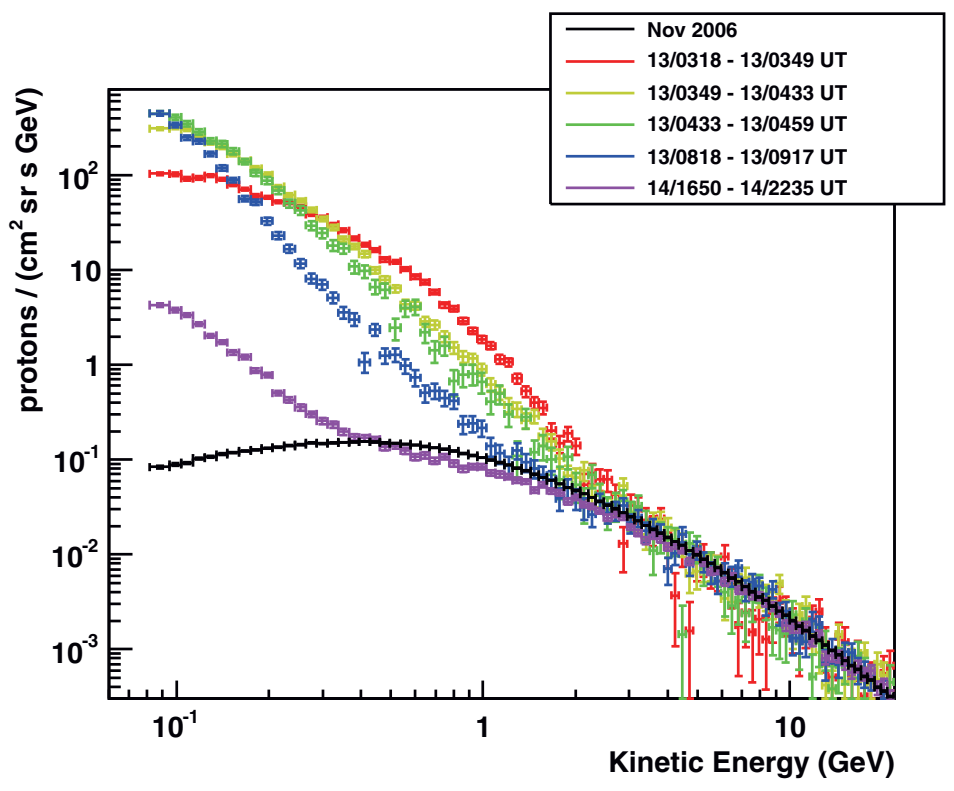

Figure 6. Measurements of p spectra performed with PAMELA during the SEP event of 13 December, 2006.

of radiation dose rates and particle background on board of low-Earth-orbit (LEO) satellites, interaction cross sections of cosmic-rays in the atmosphere and related contribution to muon and neutrino fluxes registered on ground.

The PAMELA collaboration performed [26] precision measurements of the under-cutoff ${ }^{4}$ proton fluxes with kinetic energy $>70 \mathrm{MeV}$, specifically extending the observational range of trapped proton fluxes down to low altitudes $\left(\mathrm{L}-\right.$ shell $\left.^{5} \approx 1.05\right)$ and up to the highest kinetic energies, corresponding to the trapping limit $(\approx 4 \mathrm{GeV})$, with a significant improvement of the description of the LEO radiation environment where current models suffer from the largest uncertainties.

Besides, with the PAMELA instrument it has been possible to discover [27] the presence of trapped $\bar{p}$ in the South-Atlantic anomaly ${ }^{6}$, by measuring a trapped $\bar{p}$ flux $\approx 10^{3}$ times greater than the one of galactic $\overline{\mathrm{p}}$ in the same kinetic energy range (from 60 to $750 \mathrm{MeV}$ ).

\section{Conclusions}

The PAMELA experiment is operating since 7 years on satellite, with more than $10^{9}$ triggers registered and about 30 TByte of accumulated data on incoming charged particles, achieving several important

\footnotetext{
${ }^{4}$ This term means that the proton energy is below the minimum value needed for a cosmic-ray p to reach a particular geomagnetic latitude, because of the deflection operated by the terrestrial magnetic field; therefore, under-cutoff p are necessarily originated in the Earth magnetosphere.

${ }^{5}$ The L-shell number describes the set of geomagnetic field lines which cross the Earth magnetic equator at L-shell Earth radii (in a dipole approximation of the geomagnetic field).

${ }^{6}$ Because the geomagnetic dipole axis is inclined by $\approx 11^{\circ}$ with respect to the Earth rotation axis, the inner Van Allen radiation belt comes closest to the Earth surface in correspondence of the region known as South-Atlantic anomaly (SAA), which is traversed by PAMELA during its orbit.
} 
scientific results which affect cosmic-ray physics, astrophysics and knowledge of solar-terrestrial environment. The following list summarizes the most interesting achievements of PAMELA.

- High-precision measurements of cosmic-ray antiproton/proton flux ratio and antiproton spectrum in the kinetic energy range from $60 \mathrm{MeV}$ to $180 \mathrm{GeV}$.

- First ever clear evidence that the high-energy (>10 GeV) positron fraction increases significantly (and unexpectedly) with energy.

- High-precision measurements of $\mathrm{H}$ and He nuclei spectra up to $1.2 \mathrm{TV}$ rigidity, challenging the current assumptions on cosmic-ray acceleration and propagation.

- Unique continuous study of solar modulation effects over more than 3 years of solar activity minimum.

- Precision study of trapped particles in the Earth magnetosphere, including the discovery of an antiproton radiation belt.

\section{References}

[1] P. Picozza et al., Astropart. Phys. 27, 296-315 (2007)

[2] O. Adriani et al., Science 332, 6025, 69-72 (2011)

[3] P. Blasi and E. Amato, JCAP01 10, 1-28 (2012)

[4] M.A. Malkov et al., Phys. Rev. Lett. 108, 081104, 1-5 (2012)

[5] A.E. Vladimirov et al., Astrophys. J. 752, 68, 1-19 (2012)

[6] S. Haino et al., Proc. of $33^{\text {rd }}$ ICRC, 1265, 1-4 (2013)

[7] V. Choutko et al., Proc. of $33^{\text {rd }}$ ICRC, 1262, 1-4 (2013)

[8] O. Adriani et al., Nature 458, 607-609 (2009)

[9] N.J. Shaviv et al., Phys. Rev. Lett. 103, 111302, 1-4 (2009)

[10] R. Cowsik and B. Burch, Phys. Rev. D 82, 023009, 1-7 (2010)

[11] L. Bergström et al., Phys. Rev. D 78, 103520, 1-6 (2008)

[12] I. Cholis et al., Phys. Rev. D 80, 123518, 1-19 (2009)

[13] H. Yüksel et al., Phys. Rev. Lett. 103, 051101, 1-4 (2009)

[14] P. Blasi and E. Amato, Proc. of the First Session of the Sant Cugat Forum on Astrophysics, (Springer, 2011) 623-641

[15] D. Hooper and W. Xue, Phys. Rev. Lett. 110, 041302, 1-5 (2013)

[16] O. Adriani et. al, Phys. Rev. Lett. 111, 081102, 1-6 (2013)

[17] O. Adriani et al., Phys. Rev. Lett. 102, 051101, 1-5 (2009)

[18] O. Adriani et al., Phys. Rev. Lett. 105, 121101, 1-5 (2010)

[19] M. Cirelli and G. Giesen, JCAP04 015, 0-17 (2013)

[20] B. Coste et al., Astron. Astrophys. 539, A88, 1-16 (2012)

[21] O. Adriani et al., Astrophys. J. 770, 2, 1-9 (2013)

[22] GALPROP: galprop.stanford.edu

[23] O. Adriani et al., Astrophys. J. 765, 91, 1-8 (2013)

[24] L. Maccione, Phys. Rev. Lett. 110, 081101, 1-5 (2013)

[25] O. Adriani et al., Astrophys. J. 742, 102, 1-11 (2011)

[26] A. Bruno et al., Proc. of $33^{\text {rd }}$ ICRC, 0292, 1-4 (2013)

[27] O. Adriani et al., Astrophys. J. Lett. 737, L29, 1-5 (2011) 